\title{
Insights from Pulsating Nova Envelopes
}

\author{
K. Schenker \\ Theoretical Astrophysics Group, University of Leicester, Leicester, \\ LE1 7RH, UK, e-mail: kjs@star.le.ac.uk \\ Astronomisches Institut der Universität Basel, Venusstrasse 7, $\mathrm{CH}-4102$ \\ Binningen, Switzerland
}

\begin{abstract}
Based on a linear and non-linear study of radial pulsations in the envelopes of classical novae (Schenker, 1999), I discuss the results both from the point of view of pulsation theory as well as their consequences for current nova models. Starting from initially static envelope structures at various stages during the decline of a nova outburst, strong 'running-wave' instabilities have been found that rapidly grow into shocks. Improved analytical concepts give a new direction to the interpretation of such highly non-adiabatic radial pulsations. For direct observational confirmation, a search for short period variability in the UV and soft X-ray is suggested during the very late decline phase. Speculative consequences for mass-loss scenarios in novae due to these instabilities will need some more work in the future.
\end{abstract}

\section{Introduction}

Classical nova outbursts take place in mass transferring binaries, when hydrogenrich material is accreted onto a white dwarf (WD). Once enough matter has accumulated, nuclear burning sets in explosively causing a complex and dynamical initial phase of the nova outburst. Around visual maximum however, a much more steady phase begins, where an extremely large ratio of luminosity to mass occurs and the structure of the star resembles that of a giant: a nuclear burning shell sitting on top of the WD, covered by an extended envelope of very little mass (typically $<10^{-4} M_{\odot}$ ).

Schenker (1999) analyses the pulsational stability of such a structure assuming a static envelope, or (given the ongoing evolution of the nova on time-scales of maybe weeks or months) a sequence of quasi-static envelopes.

\section{Models and results}

The background models for the linear analysis have been constructed by a linear series of core-plus-envelope models, obtained via a shooting method. Fig. 1a (left panel) shows their position in the HR diagram. Along these curves a radial, non-adiabatic linear stability analysis has been performed utilising the Riccati method (Gautschy \& Glatzel, 1990). For the 20 models specifically marked, I 

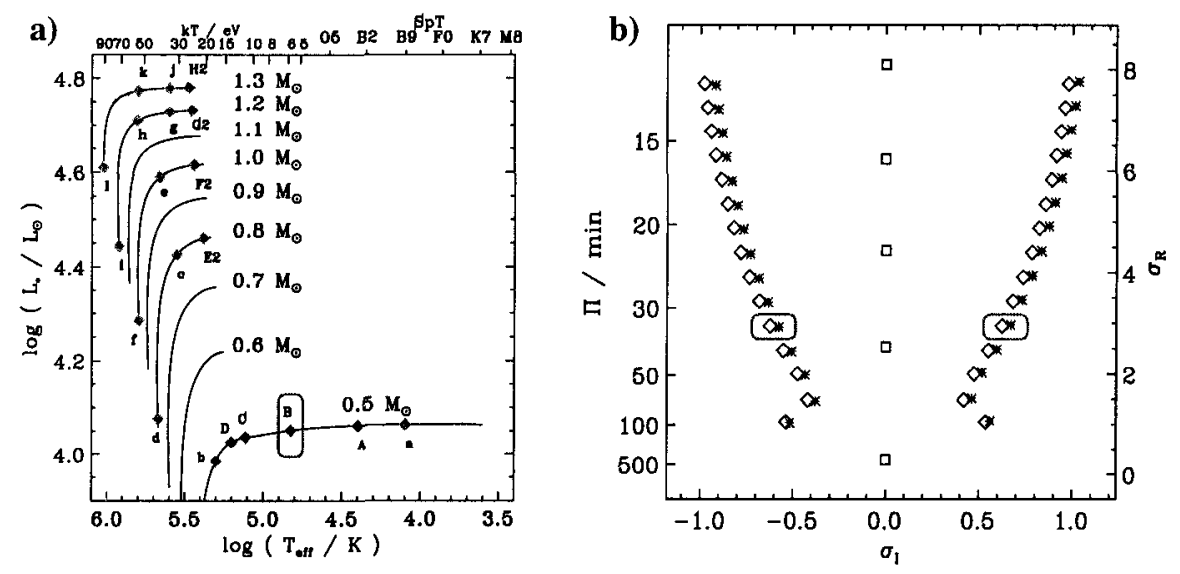

Figure 1. a) The quasi-evolutionary sequences and the specific 20 models analysed - b) Complex eigenspectrum $\left(\sigma_{\mathrm{R}}, \sigma_{\mathrm{I}}\right)$ of model B in different numerical treatment: squares adiabatic, diamonds NAR, asterisks non-adiabatic.

have also studied the non-linear evolution with adaptive-grid radiation hydrocode (RHD, Dorfi \& Feuchtinger, 1991).

One major result is plotted in Fig. $1 \mathrm{~b}$ (right panel), showing the complex eigenspectrum of model $\mathrm{B}\left(M=0.5 M_{\odot}, \log \left(T_{\text {eff }} / \mathrm{K}\right)=4.8\right)$. Frequencies $\sigma$ are plotted normalised with the free-fall time-scale in full non-adiabatic treatment and two different approximations. Most striking are the enormous growth rates found, of the same order as the free-fall time-scale. This is the case not only in model B, but for an extended range of $T_{\text {eff }}$. In contrast to classical pulsators on the instability strip (e.g., RR Lyr stars), here the adiabatic approximation cannot reproduce the frequencies at all. However, a highly suitable approximation for such a situation is the Non-Adiabatic Reversible approximation (NAR), also used by Glatzel (1994) and Saio et al. (1998) in their investigations into the nature of strange modes. One typical characteristic is the occurrence of complex conjugate pairs of eigenfrequencies, as apparent in Fig. 1b.

NAR represents the eigenmodes almost perfectly around the centre of the instability region (model B), not only in frequency (Fig. 1b) but also in terms of the eigenfunctions. These eigenfunctions (Fig. 2) represent complex standing waves, but in their projection running waves (in $x, l$, and $d$ ): outwards for the unstable, and inwards for the stable mode of each pair. In Fig. 2, fully nonadiabatic eigenfunctions are shown by amplitude and phase, so perfectly complex conjugate pairs of waves running inwards \& outwards would have identical arguments but negative phase in each of the eigenfunctions. This is almost fulfilled and a mathematical requirement for NAR solutions, with the exception of the luminosity perturbation $l$ which is effectively set to zero in this approximation. 

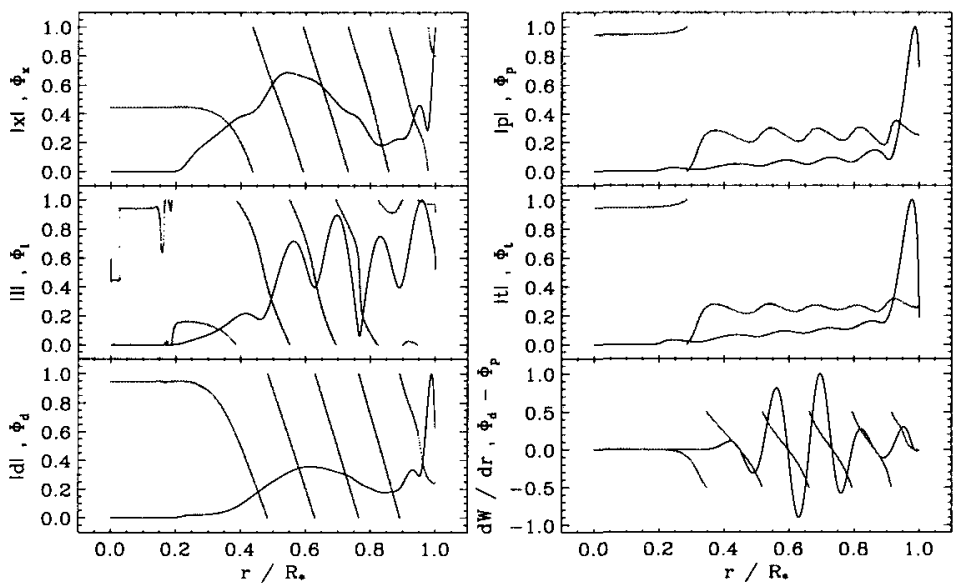

Figure 2. Set of fully non-adiabatic, complex eigenfunctions for the unstable mode of model B with $\left(\sigma_{\mathrm{R}}, \sigma_{\mathrm{I}}\right)=(2.92,-0.57)$, i.e. from the 5 th pair in Fig. 1b. In the first 5 panels, the relative Lagrangian perturbations of radial displacement $x$, luminosity $l$, density $d$, pressure $p$, and temperature $t$ are shown split into amplitude and phase angle. The last panel shows the phase difference between density and pressure perturbation together with the differential work integral.

\section{Interpretation and observational consequences}

An improved local analysis with gradients on such models reveals their nature as radiation-modified acoustic modes. It also allows to understand the spectrum found (e.g. in Fig. 1) to some degree.

The complementary analysis using the RHD code provides further characteristics of the modes and consequences in the non-linear regime. During the linear growth, and more so later on, an additional energy transport is observed, as packets of radiation energy are spiralling outwards in density perturbations (similar to a "screw conveyor"). This is likely to be responsible for the new "equilibrium" structure of background model, which actually forms a curve on the HR diagram which can significantly deviate from the initial position.

As a possibly related phenomenon with non-radial behaviour of envelopes under strong radiation fields, Shaviv (2001) has studied the application of SuperEddington winds on novae. In his model spatial variations of opacity allow a larger radiation flux to pass, whereas in the 1-dimensional case here only temporal modulations provide a similar effect.

Although the project initially attempted to explain oscillation during the so-called "transition phase" (Bianchini et al., 1992), a more probably scenario for direct observational evidence lies in the predicted X-ray oscillations shortly before the turn-off of many novae (including the faster ones on more massive WDs, Fig. 1a). 


\section{Conclusions}

It has become evident that static equilibrium models of novae after visual maximum are subject to violent pulsational instabilities. This finding (i) may suggests a possible link between mass loss in novae and pulsations, but (ii) at least excludes a purely quasi-static evolution even for the lowest mass novae (possibly systems like V723 Cas and HR Del), where common mass loss mechanisms tend to fail. For more massive WDs, only the very late phases of the outburst could be modelled. While they do also show instabilities, they still might pulsate even during the non-static earlier part.

Classical novae form an excellent laboratory for pulsations under radiation dominated conditions, so both fields will benefit from their further detailed study.

Acknowledgments. This work was financially supported by the Swiss National Science Foundation.

\section{References}

Bianchini, A., Friedjung, M., \& Brinkmann, W. 1992, A\&A, 257, 599

Dorfi, E. A. \& Feuchtinger, M. U. 1991, A\&A, 249, 417

Gautschy, A., \& Glatzel, W. 1990, MNRAS, 245, 154

Glatzel, W. 1994, MNRAS, 271, 66

Saio, H., Baker, N. H., \& Gautschy, A. 1998, MNRAS, 294, 622

Schenker, K. 1999, PhD Thesis, Universität Basel

Shaviv, N. J. 2001, MNRAS, 326, 126

\section{Discussion}

A. Cox : Is there any convection included in your calculations?

$K$. Schenker : I do not believe that convective effects are very important in this case. These envelopes are strongly dominated by radiation, and the radiative flux in the static models (which do include convection via MLT) is indeed much higher that its convective counterpart. So the linear analysis neglects the contribution of the convective flux altogether, which is consistent with the RHD models that do not contain convection at all.

A. Cox : What is the temperature of the pulsating driving region?

K. Schenker : The driving temperature lies in the range of the 'iron peak' present in the new OPAL opacities, around $10^{5.2} \mathrm{~K}$. 